\title{
Variability of sea ice deformation rates in the Arctic and their relationship with basin-scale wind forcing
}

\author{
A. Herman ${ }^{1}$ and O. Glowacki ${ }^{1,2}$ \\ ${ }^{1}$ Institute of Oceanography, University of Gdańsk, Pilsudskiego 46, 81-378 Gdynia, Poland \\ ${ }^{2}$ Department of Polar Research, Institute of Geophysics Polish Academy of Sciences, Ksiecia Janusza 64, \\ 01-452 Warsaw, Poland
}

Correspondence to: A. Herman (oceagah@ug.edu.pl)

Received: 24 July 2012 - Published in The Cryosphere Discuss.: 9 August 2012

Revised: 29 November 2012 - Accepted: 6 December 2012 - Published: 20 December 2012

\begin{abstract}
The temporal variability of the moments of probability distribution functions (pdfs) of total sea ice deformation rates in the Arctic is analyzed in the context of the basin-scale wind forcing acting on the ice. The pdfs are estimated for 594 satellite-derived sea ice deformation maps from 11 winter seasons between 1996/1997 and 2007/2008, provided by the RADARSAT Geophysical Processor System. The temporal scale analyzed equals 3 days. The moments of the pdfs, calculated for a range of spatial scales $(12.5-900 \mathrm{~km})$, have two dominating components of variability: a seasonal cycle, with deformation rates decreasing throughout winter towards a minimum in March; and a shortterm, synoptic variability, strongly correlated with the areaaveraged magnitude of the wind stress over the Arctic, estimated based on the NCEP-DOE Reanalysis-2 data (correlation coefficient of 0.71 for the mean deformation rate). Due to scaling properties of the moments, logarithms of higher moments are strongly correlated with the wind stress as well. Exceptions are observed only at small spatial scales, as a result of extreme deformation events, not directly associated with large-scale wind forcing. By repeating the analysis within regions of different sizes and locations, we show that the wind-ice deformation correlation is largest at the basin scale and decreases with decreasing size of the area of study. Finally, we suggest that a positive trend in seasonally averaged correlation between sea ice deformation rates and the wind forcing, present in the analyzed data, may be related to an observed decrease in the multi-year ice area in the Arctic, indicating possibly even stronger correlations in the future.
\end{abstract}

\section{Introduction}

Sea ice deformation constitutes an important factor in the evolution of the sea ice cover at all temporal and spatial scales. Through a number of feedbacks and interactions with other processes, it influences the ice thickness distribution, its mechanical strength, new ice production and melting, and the ocean-atmosphere heat transport.

Deformation in a compact ice pack occupying the central part of the Arctic basin is highly localized (Schulson, 2004; Stern and Lindsay, 2009). It takes place in narrow, elongated zones separating semi-rigid floes (Fig. 1a, b). Several recent studies, based on satellite data and/or drifting-buoy analysis, have revealed an intermittent, multifractal character of ice deformation (e.g., Weiss, 2001, 2008; Marsan et al., 2004; Weiss and Marsan, 2004; Rampal et al., 2009b; Stern and Lindsay, 2009; Hutchings et al., 2011). Probability distribution functions (pdfs) of deformation rates are heavy-tailed, and their tails can be well approximated by a power law. Different values of the exponents of the power-law tails have been reported, and at present no theory exists that would explain the observed variability of shapes of the pdfs of sea ice deformation rates. The intrinsic features of sea-ice deformation are generally poorly resolved in numerical sea ice models, especially those based on various versions of the viscousplastic rheology. For example, Girard et al. (2009) showed recently that sea-ice models in which deformation is based on continuum mechanics generally do not reproduce scaling properties of sea-ice deformation. Only recently, successful attempts have been made to incorporate elasto-brittle effects, crucial for long-range damage propagation, in sea ice models 
(Girard et al., 2011). However, our knowledge concerning the underlying mechanisms governing sea ice deformation still remains far from satisfactory.

Relationships between sea ice motion and deformation, on the one hand, and various components of the atmospheric and oceanic forcing, on the other hand, have been investigated in a number of studies that searched for statistically relevant correspondence between sets of variables representing the two groups of processes. Among the atmospheric variables analyzed are geostrophic wind speed (Thorndike and Colony, 1982; Serreze et al., 1989); atmospheric circulation indices, e.g., the Arctic oscillation (Kwok, 2006; Rampal et al., 2009a; Comiso, 2012); sea surface pressure distribution (Asplin et al., 2009; Kwok and Cunningham, 2011); or the number, intensity and tracks of cyclones over the Arctic, and the related cloud cover (Screen et al., 2011). The conclusions from those studies strongly depend on the type of data used (satellite, buoys, etc.), the temporal and spatial scale of the analysis, and the study period, underlining the very complicated nature of relationships and feedbacks between the atmosphere, ocean and ice processes involved.

The goal of this paper is twofold. Firstly, we want to analyze temporal variability of the properties of pdfs of observed sea ice deformation rates at temporal scales of a few days and at spatial scales from tens to hundreds of kilometers. Secondly, we want to gain insight into relationships between the wind forcing acting on the ice and ice deformation rates. To this end, we analyze short-term, synoptic variability of satellite-derived sea ice deformation rates in the Arctic basin in the context of, arguably, one of the simplest atmospheric variables conceivable, namely the area-averaged magnitude of the wind stress, calculated from the $10-\mathrm{m}$ wind speed over the Arctic basin. We demonstrate that, regardless of the fact that by performing area-averaging we lose all information on the spatial variability of the wind field, the mean wind stress still explains a substantial part of the variance of sea ice deformation rates. In the last part of the paper, we discuss the influence of the size and location of the study area on the resulting correlations, and we speculate on the meaning of our results in light of the recent climate change in the Arctic.

The paper is structured as follows: the next section contains a brief description of the data and the statistical methods used. The results of the analysis are presented in Sect. 3, followed by a discussion and conclusions in Sect. 4.

\section{Data and methods}

\subsection{Sea ice deformation and wind data}

We use the RADARSAT Geophysical Processor System (RGPS) sea ice data from the Synthetic Aperture Radar (SAR) imagery of the Arctic Ocean, provided by the RADARSAT-1 satellite. The available products include the ice motion, obtained with a feature-tracking procedure of
Kwok et al. (1990). The RGPS products have a temporal resolution of 3 days. The data are available in two versions: in a Lagrangian form, as well as processed onto a regular grid in a polar stereographic projection, with a constant spatial resolution $\Delta x=12.5 \mathrm{~km}$ (see http://rkwok.jpl.nasa.gov/radarsat/ 3daygridded.html). In this work, we use the gridded RGPS fields of the shear rate $\dot{\epsilon}_{\mathrm{s}}$, and the divergence rate $\dot{\epsilon}_{\mathrm{d}}$, defined as

$$
\dot{\epsilon}_{\mathrm{d}}=\frac{\partial u}{\partial x}+\frac{\partial v}{\partial y}, \dot{\epsilon}_{\mathrm{s}}=\left[\left(\frac{\partial u}{\partial x}-\frac{\partial v}{\partial y}\right)^{2}+\left(\frac{\partial u}{\partial y}+\frac{\partial v}{\partial x}\right)^{2}\right]^{1 / 2}
$$

where $u$ and $v$ denote the velocity components along the $x$ and $y$ axes of the regular grid, respectively. At present, the available data cover 11 winter seasons (November-April) from 1996/1997 to 2007/2008 (except 2002/2003). Data from summer periods are not used in this study.

The daily $10-\mathrm{m}$ wind speed data come from the NCEPDOE Reanalysis 2 data set (Kanamitsu et al., 2002). The data are available on a global grid with a spatial resolution $1.875^{\circ}$ longitude and $1.904^{\circ}$ latitude.

\subsection{Data preprocessing and analysis}

Our analysis is limited to the total deformation rate $\dot{\epsilon}_{\mathrm{t}}$, which is a scalar quantity defined as (e.g., Girard et al., 2009)

$\dot{\epsilon}_{\mathrm{t}}=\left(\dot{\epsilon}_{\mathrm{s}}^{2}+\dot{\epsilon}_{\mathrm{d}}^{2}\right)^{1 / 2}$.

We calculated the moments $m_{q, L}$ of the pdfs of $\dot{\epsilon}_{\mathrm{t}}$ for a range of spatial scales $L=n \Delta x$, starting from the original mesh size $\Delta x(n=1)$ up to $916 \mathrm{~km}(n=73)$, and for the power $q$ ranging from 0.5 to 3.0 :

$m_{q, L}=\left\langle\dot{\epsilon}_{\mathrm{t}, L}^{q}\right\rangle$,

where $\langle\cdot\rangle$ denotes averaging over all grid cells of a given deformation-rate map with resolution $L$ (Fig. 1c). The maps with resolution lower than $\Delta x$, i.e., for $n>1$, were obtained by averaging of the original data within $n \times n$ windows and removing meshes containing fewer than $n^{2} / 2$ data points. After removal of maps with very large amount of gaps, a total of $N=594$ maps have been retained for further analysis, each with at least $1.2 \times 10^{4}$ data points.

The magnitude of wind stress $\tau_{\mathrm{a}}$ was calculated from the 10 -m wind speed $U_{10}$ as $\tau_{\mathrm{a}}=\rho_{\mathrm{a}} C_{\mathrm{D}} U_{10}^{2}$, assuming a constant air density $\rho_{\mathrm{a}}=1.27 \mathrm{~kg} \mathrm{~m}^{-3}$ and an air-ice drag coefficient $C_{\mathrm{D}}=2 \times 10^{-3}$. The time series of basin-averaged wind stress $\bar{\tau}_{\mathrm{a}}=\bar{\tau}_{\mathrm{a}}(t)$ was obtained by averaging within the region marked in Fig. 1 and over three-day periods leading the corresponding time windows of the RGPS data by $\Delta t=2$ days. To account for variable spatial resolution of the NCEP/DOE data, the values of $\tau_{\mathrm{a}}$ were interpolated onto the regular RGPS grid before averaging. The optimal value of $\Delta t$ was selected based on a preliminary analysis of correlation coefficients between $\bar{\tau}_{\mathrm{a}}$ and logarithms of $m_{q, L}$ (see further). 
(a)

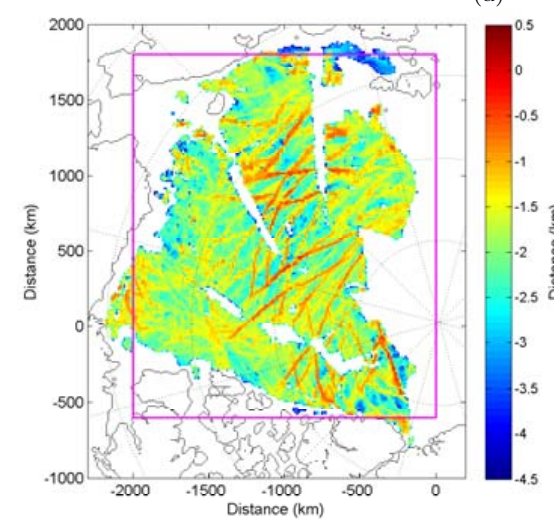

(b)

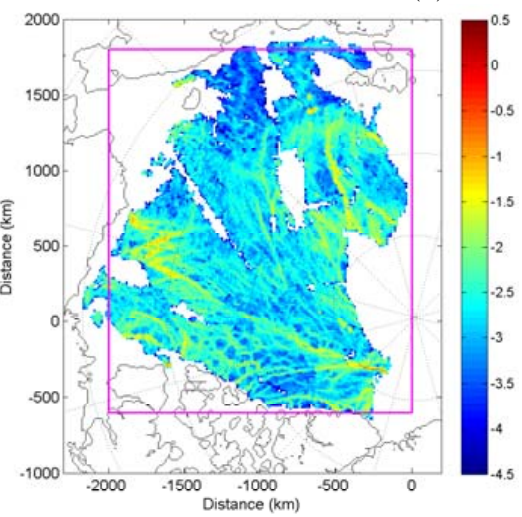

(c)

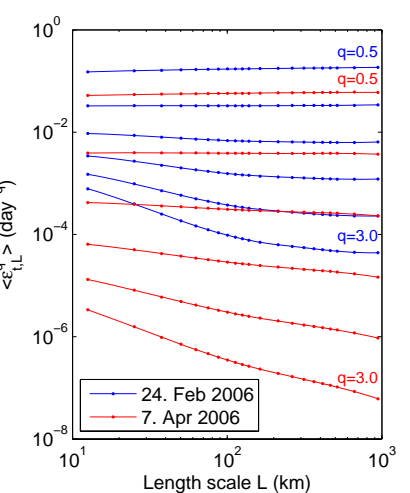

Fig. 1. Total sea ice deformation rate $\dot{\epsilon}_{\mathrm{t}}$ in the Arctic on 24 February $\left(\bar{\tau}_{\mathrm{a}}=0.24 \mathrm{~N} \mathrm{~m}^{-2}\right)$ and 7 April $2006\left(\bar{\tau}_{\mathrm{a}}=0.03 \mathrm{~N} \mathrm{~m}^{-2}\right)$ : maps of $\dot{\epsilon}_{\mathrm{t}}($ in day $^{-1}$ ), shown in a logarithmic color scale (a,b); and moments $m_{q, L}$ for a range of length scales $L$ and powers $q$ (c). The boxes in (a) and (b) show the area within which $\bar{\tau}_{a}$ was calculated. The lines in (c) are drawn for $q$ increasing from 0.5 to 3.0 with a step of 0.5 .

All correlation coefficients reported in the remaining part of this paper were calculated with a bootstrap method by averaging over 1000 random sub-samples of the original data sets, each containing $99 \%$ of all data points. De Boor's cubic smoothing spline method (de Boor, 2001) was used to estimate nonlinear trends in the ice deformation data. The smoothing factor was set by trial and error to $5 \times 10^{-5}$ (the correlations presented further were not sensitive to this choice as long as this parameter was lower than $1 \times 10^{-4}$ ).

\section{Results}

Figure 1 shows two representative examples of sea ice deformation rate maps from the analyzed data set. As is already known (see, e.g., Marsan et al., 2004; Hutchings et al., 2011), deformation rates have multifractal properties, with

$m_{q, L} \sim L^{-\beta(q)}$,

where $\beta$ is a positive, increasing, convex function of $q$. For mean sea ice deformation rates $(q=1)$ in the Arctic, $\beta$ is close to 0.2 (Marsan et al., 2004), although it exhibits a clear seasonal cycle with highest values during the summer (Stern and Lindsay, 2009). Generally, deviations from the scaling relationship occur only for higher moments $(q>2)$ and small spatial scales during short episodes of strong deformation, like the one shown in Fig. 1a and c. Similar to the exponent $\beta$, the mean magnitude of sea ice deformation reveals a pronounced seasonal and short-term time variability, reflecting the state of the ice cover and the forcing acting on it.

Let us introduce the notation: $\tilde{m}_{q, L} \equiv \log _{10} m_{q, L}$. From the scaling relationship (Eq. 4), it follows that

$\tilde{m}_{q_{1}, L_{2}}=\tilde{m}_{q_{1}, L_{1}}-\beta\left(q_{1}\right) \log _{10}\left(L_{2} / L_{1}\right)$ and

$\tilde{m}_{q_{2}, L_{1}} \sim \frac{\beta\left(q_{2}\right)}{\beta\left(q_{1}\right)} \tilde{m}_{q_{1}, L_{1}} ;$

i.e., the relationship between the logarithms of the moments at different scales $L$ and for different powers $q$ is linear.

In the following, the correlation coefficient between $\bar{\tau}_{\mathrm{a}}$ and $\tilde{m}_{q, L}$ will be denoted with $C=C(q, L)$. It must be noticed that the range of values of $m_{1, L}$ in the RGPS data set, between 0.003 and $0.044 \mathrm{day}^{-1}$, is small enough so that $\tilde{m}_{1, L}$ can be approximated as a linear function of $m_{1, L}$ - the respective correlation coefficient equals 0.965 , and correlation between $\bar{\tau}_{\mathrm{a}}$ and $m_{1, L}$ equals 0.73 . The reason for using the logarithm $\tilde{m}_{1, L}$ instead of non-logarithmic values of $m_{1, L}$ is for consistency of the treatment of all moments, for which the scaling relationships Eqs. (5) and (6) are expected to hold.

As mentioned in Section 2.2, the values of $C$ are highest for a time lag $\Delta t$ between the two data sets equal to 2 days. Presumably, the optimal value of $\Delta t$ is smaller in weaker first-year ice, reacting faster to changes of the external forcing than in thick and strong multi-year ice; however, as this study concentrates on the basin-scale forcing and sea ice deformation, this supposed spatial variability of the characteristic reaction time of the ice cover to the forcing is not taken into account and $\Delta t$ is set constant. Figure 2a shows a scatter plot of the data for $q=1$ and $L=\Delta x$ (scatter plots for other $(q, L)$-combinations are similar). As expected, the deformation rates increase with increasing wind forcing, with $C=0.61$ for the mean deformation rate $(q=1)$ and slightly lower values for higher moments (not shown). However, the distribution of the data points reveals an interesting seasonal pattern of sea ice deformation. The same mean wind stress tends to be associated with higher deformation rates at the beginning of the winter season (November-December) than in late winter and early spring (March-April). A seasonal cycle, with a tendency of the mean deformation rates to decrease 
(a)
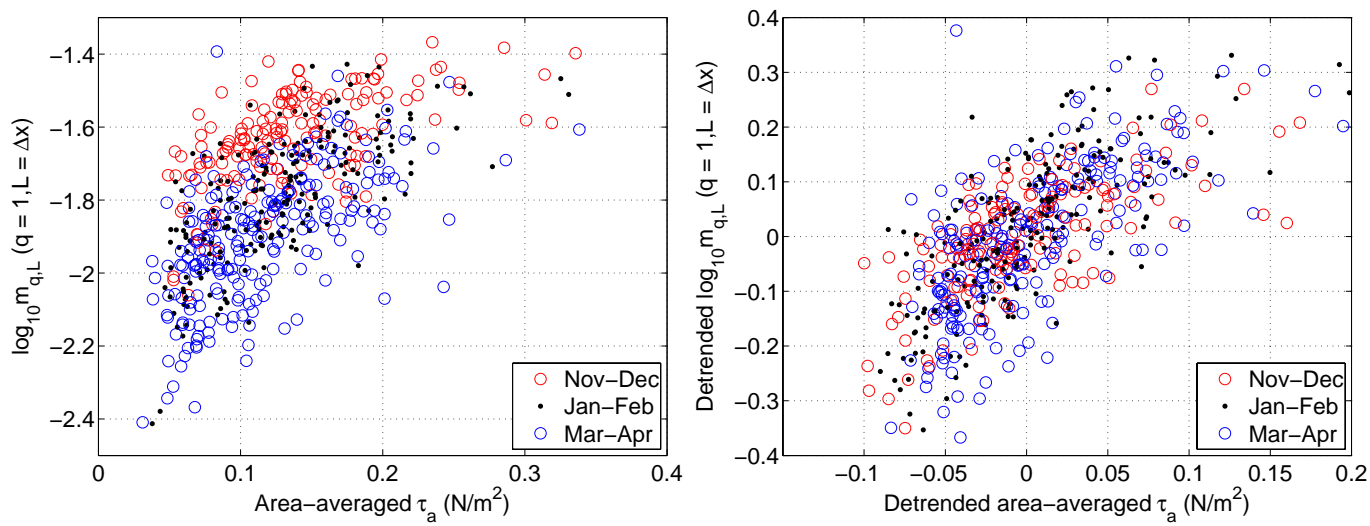

Fig. 2. Scatter plots of $\tilde{m}_{q, L}$ (for $q=1$ and $L=\Delta x$ ) against $\bar{\tau}_{a}$ for the original $(\mathbf{a} ; C=0.61)$ and seasonally detrended $(\mathbf{b} ; C=0.71)$ data from the whole analysis period 1996-2008.

from November to March, is clearly visible in the time series of $\tilde{m}_{q, L}$, shown in Fig. 3a for the 1999/2000 winter, and in the Supplement Fig. 1 for the remaining winter seasons. Although a strong inter-annual variability is present, nonlinear trend lines fitted to the data within individual seasons generally have a minimum in March, coinciding with the maximum thickness and extent of the ice cover. The trend changes sign in April, with the onset of the melting season. Not surprisingly, this intra-seasonal variability is in agreement with the annual cycle of the mean monthly shear rates reported by Kwok (2006) and Kwok and Cunningham (2011), as well as the annual cycle of mean deformation rates obtained by Stern and Lindsay (2009), who used the Lagrangian RGPS data. Similarly, there is a clear annual minimum in March in drifting-buoy-derived sea ice speeds calculated by Rampal et al. (2009a). Strong seasonal variability in mechanical behavior of the Arctic sea ice has been also found by Gimbert et al. (2012a,b), who analyzed changes of the inertial motion intensity in the Arctic.

The correlation coefficients $C$, recalculated for seasonally detrended data, can be significantly higher than those obtained for the original time series (Figs. $2 b$ and $3 b$ ). Generally, if the deformation data exhibited perfect scaling, $C(q, L)$ would be constant due to the linear relationships in Eqs. (5) and (6). Thus, the variability of $C(q, L)$, shown in Fig. 4 , mirrors the deviations from the scaling properties of the moments. For instance, for low values of $q$ the deviations from scaling are small (Fig. 1c), and hence no strong dependence of $C$ on $L$ can be observed, with $C$ decreasing slowly with $L$ from 0.71 to 0.68 . The lowest values of $C$, still statistically significant (at a $99 \%$ confidence level), occur only for high moments and small length scales, i.e., for $(q, L)$ combinations for which the largest deviations from scaling Eq. (6) are present. Clearly, as the higher moments are very sensitive to exceptionally large values, those $(q, L)$-pairs represent mainly very strong, localized deformation events.
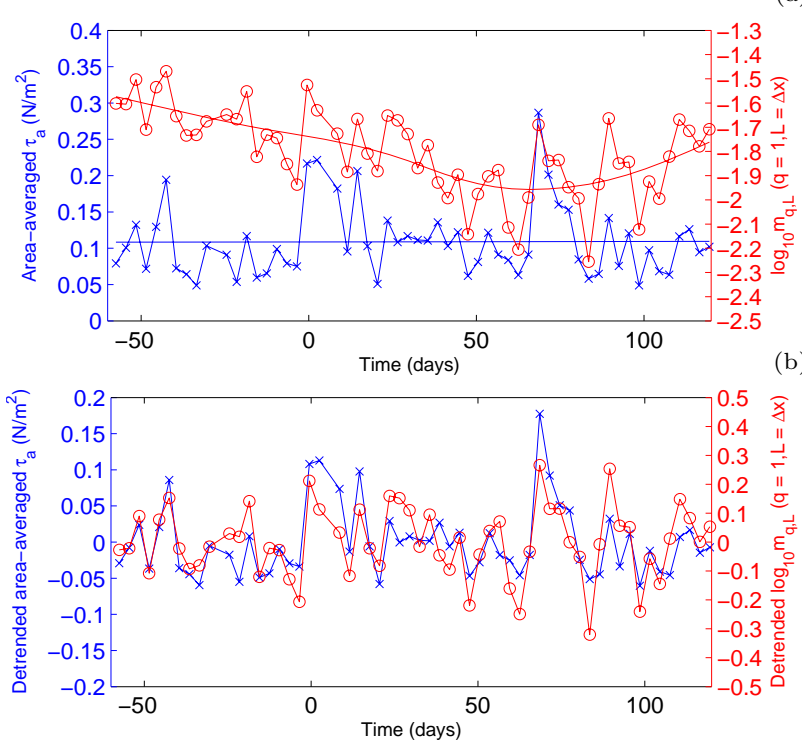

Fig. 3. Time series of $\bar{\tau}_{\mathrm{a}}\left(\mathrm{Nm}^{-2}\right)$ and $\tilde{m}_{q, L}$ (for $q=1$ and $L=$ $\Delta x$ ) in the 1999/2000 winter: original data with trend lines (a) and detrended data (b). Time is shown in days after 1 January 2000. See Supplement Fig. 1 for other winter seasons.

The occurrence probability of those events is not uniform in space, but is significantly higher in some coastal regions, especially along the coast of Alaska, in the East Siberian Sea and close to the New Siberia Islands, as shown in Fig. 5. Interestingly, there are no corresponding occurrence maxima along the coast of the Canadian Archipelago - presumably because of the larger thickness and strength of the (predominantly perennial) ice pack in that region. As shown in a number of studies (e.g., Steele et al., 1997; Kwok, 2006), seasonal sea ice in the Beaufort and East Siberian Sea has lower thickness and concentration, and higher velocities than 


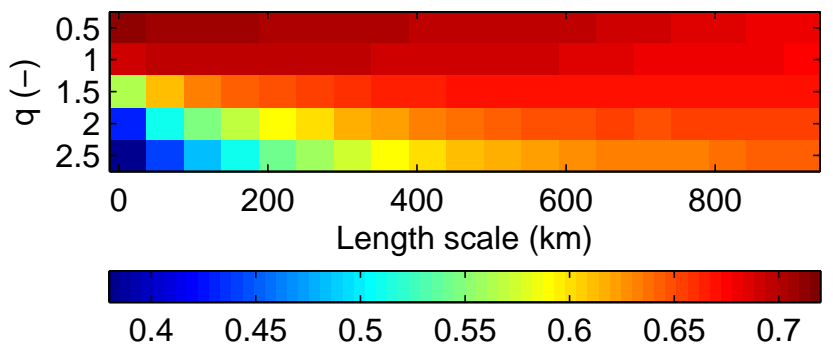

Fig. 4. Variability of the correlation coefficient $C(q, L)$ between $\bar{\tau}_{\mathrm{a}}$ and $\tilde{m}_{q, L}$, calculated for seasonally detrended data from the whole data set.

elsewhere, which makes it susceptible to coast-constrained deformation.

Considering the inhomogeneous spatial distribution of strong-deformation events, it is natural to repeat the above correlation analysis separately for two subdomains of the study area - loosely termed the "coastal" and the "central" region (including the coastal zone of Canada). As Fig. 5 schematically illustrates, the subdomains were obtained by stepwise moving the upper-left corner of the original domain towards its center. At each step, the correlation $C$ was calculated for the two regions separately (i.e., for $\bar{\tau}_{\mathrm{a}}$ and $\tilde{m}_{q, L}$ ) estimated based on a subset of data from that region. The results for $L=\Delta x$ and two selected values of $q$ are shown in Fig. 6. The values of $C$ increase with the increasing width of the coastal region, especially within $\sim 400 \mathrm{~km}$ from the coast (circles in Fig. 6) - remarkably, the same distance was found by Thorndike and Colony (1982) as the boundary of the area within which the coasts influence the sea ice motion. This seems to favor the line of reasoning sketched above. However, within the central region, the values of $C$ for $q=1$ do not increase, but decrease with increasing distance of its edges from the coast, i.e., with decreasing surface area of that region. The rate of change of $C$ with changing surface area is similar in the two domains, suggesting that the size of the area of study has more influence on $C$ than the location of that area within the Arctic Basin (tests for subdomains with randomly selected positions and sizes - not shown - confirm this conclusion). This aspect of the results is discussed further in the next section.

\section{Discussion and conclusions}

The use of the surface wind stress, i.e., a quantity directly determining forcing acting on the ice - as opposed to other variables, e.g., the atmospheric pressure or upper-level winds, used in a number of other studies - seems particularly appropriate for the analysis of atmosphere-sea ice relationships on short time scales. This is especially true in winter, when, due to high vertical stability of the lower troposphere (e.g., Devasthale et al., 2010), there is no close coupling between the boundary layer and free-troposphere winds. This decou-

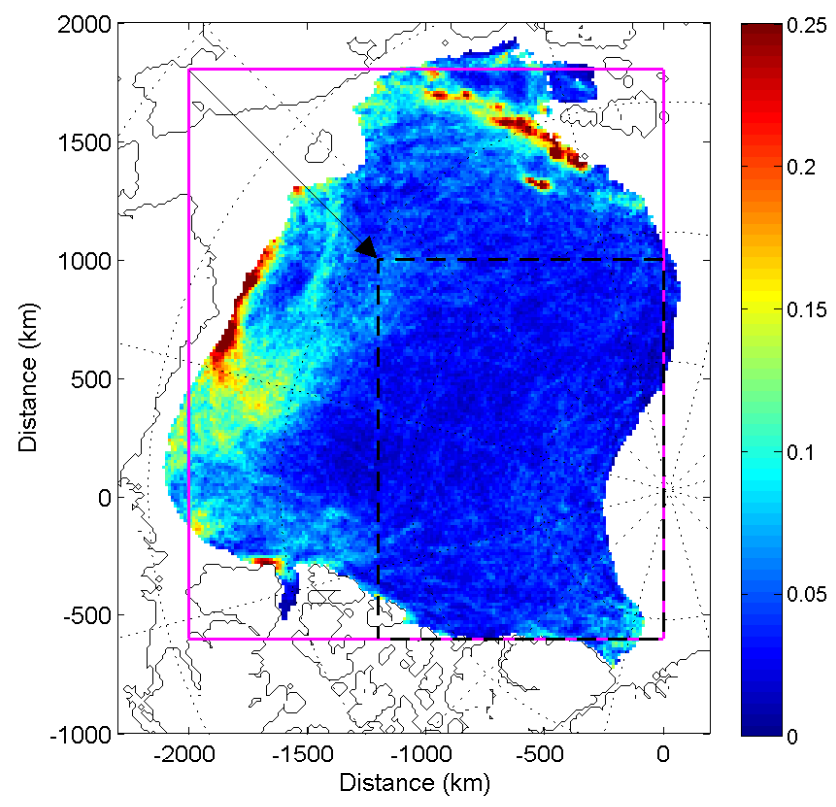

Fig. 5. Occurrence probability of extremely strong deformation events in the study area, defined as the largest $5 \%$ of all $\dot{\epsilon}_{\mathrm{t}}$ values from each deformation map. The rectangular frames and the arrow illustrate the division of the study area into the "central" and the "coastal" region (within and outside of the dashed box, respectively), used to study the influence of the domain size and location on the values of $C$ (see text for a detailed description).

pling may partly explain low correlation between sea ice deformation and indices of large-scale atmospheric circulation reported by some authors (e.g., Rampal et al., 2009a). As argued recently by Tsukernik et al. (2010), sufficient explanation of sea ice-atmosphere interactions may require accounting for processes acting on time scales shorter than monthly or seasonal.

The results described in this work suggest the existence of two important components of variability of sea ice deformation rates in the Arctic. The first is the annual cycle (Fig. 3a and Supplement Fig. 1), which accounts for $\sim 50 \%$ of the variance of the mean deformation rates (the percentage is lower for the higher moments). It does not have a relationship with wind forcing, but reflects seasonal changes of the properties of the ice cover itself - its thickness, compactness, mechanical strength and, importantly, ice-extent related degree of confinement by the basin's boundaries. Although seasonally varying momentum transfer between the atmosphere and sea ice has been proposed previously as one of the causes of the existence of a seasonal cycle in ice motion and deformation (Steele et al., 1997; Kwok, 2006), much of this "observed" influence results from the usage of geostrophic wind in those studies. The above-mentioned annual cycle in atmospheric stability, if not accounted for, produces a spurious cycle in the air-ice stress, seemingly correlated with seasonal changes in ice deformation. Figure 3 and Supplement Fig. 1 


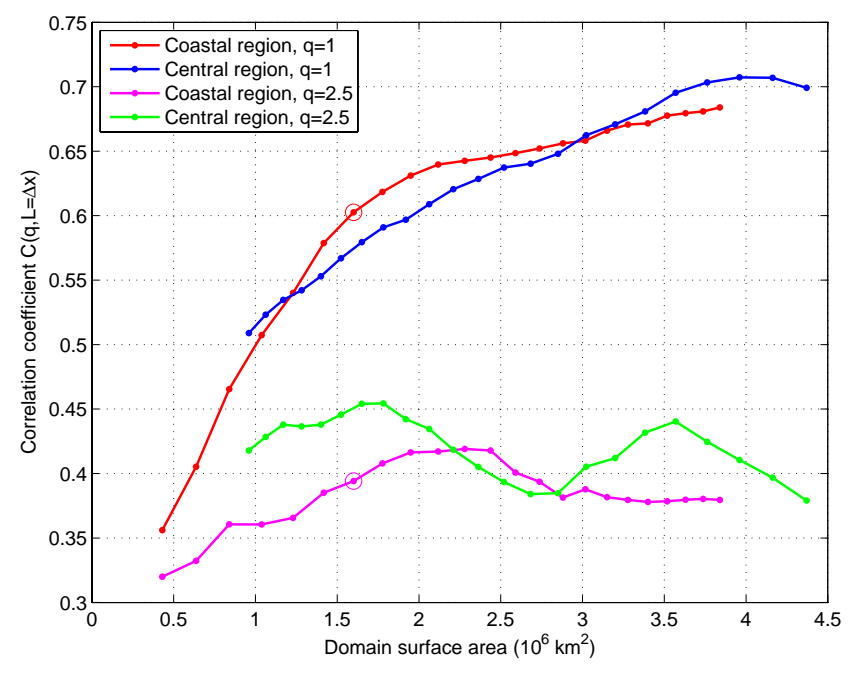

Fig. 6. Variability of $C(q, L=\Delta x)$ with changing size of the two analyzed sub-domains, the "coastal" region and the "central" region (Fig. 5), for $q=1$ and $q=2.5$. Data points corresponding to the width of the coastal region equal to $400 \mathrm{~km}$ are marked with circles.

clearly show that there is hardly any variability in the magnitude of the NCEP surface wind data on a seasonal scale.

The second component of variability is the (sub)synoptic variability related - directly or indirectly via other processes - to the wind stress acting on the ice (Fig. 3b). In the case of the mean deformation rates, changes of the area-averaged wind stress account for approximately $50 \%$ of their shortterm variance (i.e., variance of seasonally detrended time series), independently of the spatial scale. The remaining $50 \%$ can be attributed to other processes - additional forcing mechanisms (predominantly the ice-water stress, but also forces due to the sea surface tilt and the Coriolis force), nonlinear components of the sea ice rheology, as well as measurement errors. The results of this study do not allow for distinguishing between those additional factors. The importance of particular processes may vary both temporally and spatially - as demonstrated by a number of other studies of sea ice deformation in the Arctic (Steele et al., 1997) and in the Weddell Sea (Hutchings et al., 2012). It is worth noticing that - as the results described in the previous section show - the percentage of the variance of sea ice deformation explained by the wind forcing depends on the size of the domain of analysis. This finding is in agreement with the recent results of Hutchings et al. (2011), who studied sea ice deformation in the Beaufort Sea measured with an array of GPS drifters. They found that the spatial coherence displayed by sea ice deformation is low at sub-synoptic scales and increases with increasing scale of observation, and that (at least in late winter and early spring) the coherent behavior of deformation is controlled by the synoptic atmospheric forcing. The variability of $C$ shown in Fig. 6 suggests sim-

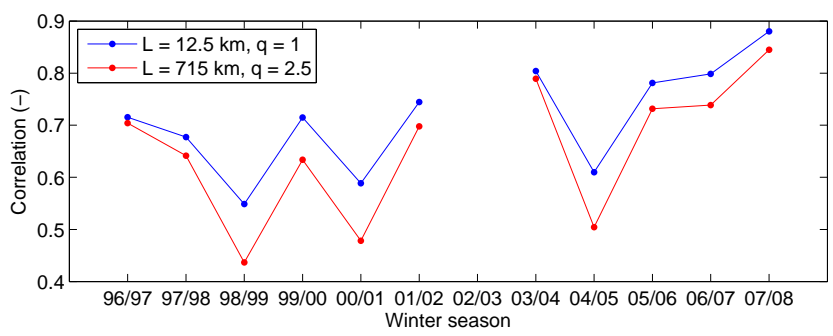

Fig. 7. Inter-annual variability of $C$ in the study period for two selected $(q, L)$-pairs, calculated for seasonally detrended data.

ilar behavior: for large $q$, i.e., moments representing strong, localized deformation, the values of $C$ remain low and relatively constant independently of the scale of observation; and for the mean deformation rates $(q=1)$, coherence with the wind forcing emerges with increasing spatial scale. Thus, our results confirm the conclusion - important for numerical modeling of sea ice - that the localized, small-scale ice deformation events, important for atmosphere-ocean heat exchange and new ice production, are not directly correlated with large-scale wind-stress patterns.

Finally, it is interesting to note a slightly positive trend in correlation between the wind forcing and sea ice deformation rates, calculated separately for individual winter seasons within the analysis period (Fig. 7). Obviously, 11 data points are far from sufficient for any statistics. Nevertheless, it is tempting to hypothesize that this trend is related to the recent decrease in the multi-year ice area in the Arctic (Comiso, 2012). Deformation rates are generally higher in seasonal, relatively weak and thin ice than in the thick, multi-year ice. First-year ice is expected to respond more directly to the wind forcing, and hence its increasing amount may lead to higher basin-scale wind stress-ice deformation correlations. Thus, higher sea ice deformation rates are expected for those winters, for which particularly strong melting occurred in the preceding summer and autumn periods. Interestingly, within the period of study there were 3 local minima in the November multi-year ice area, as estimated by Comiso (2012) - in 1999, 2007, and, less pronounced, in 2003. They correspond to the peaks in seasonally averaged values of $C$ in the following winters (Fig. 7), with the correlation coefficient for $q=1$ approaching a remarkably high value of 0.9 in the 2007/2008 winter, after the record ice minimum in the summer of 2007. Another indication of longer-term processes may be a change in the seasonal cycle of deformation that occurred during the analysis period. Comparison of the trend lines shown in Supplement Fig. 1 clearly shows that the March minimum of deformation rates has become shallower; i.e., the wintertime negative trend in deformation rates has decreased (only 2 winters, 1997/1998 and 2005/2006, show an exception from this tendency). It may be a consequence of the thinning of the ice cover, which is becoming more susceptible to deformation even during the 
maximum annual sea ice extent and thickness. This notion is supported by the results of a recent study by Gimbert et al. (2012b) (see also Gimbert et al., 2012a) who observed a positive trend in the inertial-motion intensity in the Arctic over the last decade and interpreted it as a consequence of a mechanical weakening of the ice cover. More data covering a longer time period will be necessary to verify the statistical significance of those trends.

\section{Supplementary material related to this article is available online at: http://www.the-cryosphere.net/6/ 1553/2012/tc-6-1553-2012-supplement.pdf.}

Acknowledgements. NCEP Reanalysis 2 data were provided by the NOAA/OAR/ESRL PSD, Boulder, Colorado, USA, from their Web site at http://www.esrl.noaa.gov/psd/. RGPS data are made available by the Jet Propulsion Laboratory at http://rkwok.jpl.nasa.gov/radarsat/index.html. We are grateful to Jennifer Hutchings, Florent Gimbert and an anonymous reviewer for their valuable and insightful comments on the first version of our manuscript.

Edited by: R. Lindsay

\section{References}

Asplin, M., Lukovich, J., and Barber, D.: Atmospheric forcing of the Beaufort Sea ice gyre: Surface pressure climatology and sea ice motion, J. Geophys. Res., 114, C00A06, doi:10.1029/2008JC005127, 2009.

Comiso, J.: Large decadal decline of the Arctic multiyear ice cover, J. Climate, 25, 1176-1193, 2012.

de Boor, C.: A Practical Guide to Splines (Revised Edition), Springer Verlag, 360 pp., 2001.

Devasthale, A., Willén, U., Karlsson, K.-G., and Jones, C. G.: Quantifying the clear-sky temperature inversion frequency and strength over the Arctic Ocean during summer and winter seasons from AIRS profiles, Atmos. Chem. Phys., 10, 5565-5572, doi:10.5194/acp-10-5565-2010, 2010.

Gimbert, F., Jourdain, N., Marsan, D., Weiss, J., and Barnier, B.: Recent mechanical weakening of the Arctic sea ice cover as revealed from larger inertial oscillations, J. Geophys. Res., 117, C00J12, doi:10.1029/2011JC007633, 2012a.

Gimbert, F., Marsan, D., Weiss, J., Jourdain, N. C., and Barnier, B.: Sea ice inertial oscillations in the Arctic Basin, The Cryosphere, 6, 1187-1201, doi:10.5194/tc-6-1187-2012, 2012b.

Girard, L., Weiss, J., Molines, J., Barnier, B., and Bouillon, S.: Evaluation of high-resolution sea ice models on the basis of statistical and scaling properties of Arctic sea ice drift and deformation, J. Geophys. Res., 114, C08015, doi:10.1029/2008JC005182, 2009.

Girard, L., Bouillon, S., Weiss, J., Amitrano, D., Fichefet, T., and Legat, V.: A new modeling framework for sea-ice mechanics based on elasto-brittle rheology, Ann. Glaciol., 52, 123-132, 2011.
Hutchings, J., Roberts, A., Geiger, C., and Richter-Menge, J.: Spatial and temporal characterization of sea-ice deformation, Ann. Glaciol., 52, 360-368, 2011.

Hutchings, J., Heil, P., Steer, A., and Hibler III, W. D.: Subsynoptic scale spatial variability of sea ice deformation in the western Weddell Sea during early summer, J. Geophys. Res., 117, C01002, doi:10.1029/2011JC006961, 2012.

Kanamitsu, M., Ebisuzaki, W., Woollen, J., Yang, S.-K., Hnilo, J., Fiorino, M., and Potter, G.: NCEP-DOE AMIP-II reanalysis (R2), B. Am. Meteorol. Soc., 11, 1631-1643, doi:10.1175/BAMS83-11-1631, 2002.

Kwok, R.: Contrasts in sea ice deformation and production in the Arctic seasonal and perennial ice zones, J. Geophys. Res., 111, C11S22, doi:10.1029/2005JC003246, 2006.

Kwok, R. and Cunningham, G.: Deformation of the Arctic Ocean ice cover after the 2007 record minimum in summer ice extent, Cold Reg. Sci. Technol., 76-77, 17-23, 2011.

Kwok, R., Curlander, J., McConnell, R., and Pang, S.: An icemotion tracking system at the Alaska SAR facility, IEEE J. Oceanic Eng., 15, 44-54, 1990.

Marsan, D., Stern, H., Lindsay, R., and Weiss, J.: Scale dependence and localization of the deformation of Arctic sea ice, Phys. Rev. Lett., 93, 178501, doi:10.1103/PhysRevLett.93.178501, 2004.

Rampal, P., Weiss, J., and Marsan, D.: Positive trend in the mean speed and deformation rate of Arctic sea ice, 1979-2007, J. Geophys. Res., 114, C05013, doi:10.1029/2008JC005066, 2009a.

Rampal, P., Weiss, J., Marsan, D., and Bourgoin, M.: Arctic sea ice velocity field: General circulation and turbulent-like fluctuations, J. Geophys. Res., 114, C10014, doi:10.1029/2008JC005227, 2009b.

Schulson, E.: Compressive shear faults within arctic sea ice: Fracture on scales large and small, J. Geophys. Res., 109, C07016, doi:10.1029/2003JC002108, 2004.

Screen, J., Simmonds, I., and Keay, K.: Dramatic interannual changes of perennial Arctic sea ice linked to abnormal summer storm activity, J. Geophys. Res., 116, D15105, doi:10.1029/2011JD015847, 2011.

Serreze, M., Barry, R., and McLaren, A.: Seasonal variations in sea ice motion and affects on sea ice concentration in the Canada Basin, J. Geophys. Res., 94, 10955-10970, 1989.

Steele, M., Zhang, J., Rothrock, D., and Stern, H.: The force balance of sea ice in a numerical model of the Arctic Ocean, J. Geophys. Res., 102, 21061-21079, 1997.

Stern, H. and Lindsay, R.: Spatial scaling of Arctic sea ice deformation, J. Geophys. Res., 114, C10017, doi:10.1029/2009JC005380, 2009.

Thorndike, A. and Colony, R.: Sea ice motion in response to geostrophic winds, J. Geophys. Res., 87, 5845-5852, 1982.

Tsukernik, M., Deser, C., Alexander, M., and Thomas, R.: Atmospheric forcing of Fram Strait sea ice export: A closer look, Clim. Dynam., 35, 1349-1360, doi:10.1007/s00382-009-0647-z, 2010.

Weiss, J.: Fracture and fragmentation of ice: a fractal analysis of scale invariance, Eng. Fract. Mech., 68, 1975-2012, 2001.

Weiss, J.: Intermittency of principal stress directions within Arctic sea ice, Phys. Rev. E, 77, 056106, doi:10.1103/PhysRevE.77.056106, 2008.

Weiss, J. and Marsan, D.: Scale properties of sea ice deformation and fracturing, C. R. Physique, 5, 735-751, 2004. 